\title{
SITUAÇÃO DE SAÚdE MATERNA E PERINATAL NO ESTADO DE SÃO PAULO, BRASIL
}

\author{
Ana Cristina d'Andretta Tanaka* \\ Arnaldo Augusto Franco de Siqueira* \\ Paulo Nogueira Bafile**
}

\begin{abstract}
TANAKA, A.C. d'A. et al. Situação de saúde materna e perinatal no Estado de São Paulo, Brasil. Rev. Saúde públ., S. Paulo, 23:67-75, 1989.

RESUMO: Pelos dados of iciais disponiveis, para o Estado de Såo Paulo (Brasil) analisou-se a assistência oferecida na gestação, no parto e ao recém-nascido e suas relações com a mortalidade materna e perinatal. Com referência ao pré-natal, houve indícios de quantidade de consultas por gestação, numericamente suficiente, porém de qualidade discutível. Quanto ao parto observou-se uma alta percentagem de cesarianas $(46,2 \%)$. A mortalidade materna foi de 4,86 por dez mil n.v., valor este subestimado. A primeira causa de b́bitos maternos foram as toxemias, seguidas das hemorragias e do aborto, causas estas evitáveis em sua maioria, com uma boa qualidade de assistência pré-natal e ao parto. Quanto aos óbitos do período perinatal, o coeficiente foi de 29,2 por mil nascimentos em 1984, valor este tambem elevado. A análise das causas de b́bitos mostrou que as afecçóes de origem perinatal ocorreram em cerca de $90 \%$ dos óbitos, tendo como principais causas as hipóxias intra-uterinas, asfixias, sindromes de angústia respiratória e aspiração maciça. Esses dados revelam a má qualidade de assisténcia recebida por este grupo. Sugere-se que a nova política de Sistema Unificado e Descentralizado de Saúde deveria levar em conta tanto a qualidade de assistência como a integraçăo dos serviços para poder-se fazer frente à situaçăo precária de saúde materna e perinatal do Estado.
\end{abstract}

DESCRITORES: Assistência pré-natal. Parto. Mortalidade materna. Mortalidade perinatal. Saúde materno-infantil.

\section{INTRODUÇÃO}

A situação de saúde materna e perinatal apresenta grande relevância pelo fato de, além dos aspectos quantitativos (magnitude), as mulheres e seus conceptos representaram um grupo de alta vulnerabilidade, expressa no maior risco de adoecer e morrer a que estão expostos.

Além de aspectos eminentemente biológicos, a saúde desse grupo populacional é também um reflexo muito nítido das condiçōes sócio-econômicas e assistenciais a que esta população está sujeita.

No momento atual só se consegue visualizar a real situação de saúde desse grupo através de coeficientes de mortalidade. Esses coeficientes servem também como indicadores específicos da qualidade de assistência à gestação, ao parto e ao recém-nascido. Assim, por exemplo, em países desenvolvidos como a Noruega, Suécia, Japão e EUA, em que nas últimas décadas esses coeficientes têm diminuído de valores já consideravelmente reduzidos, porém continuam diminuindo de maneira muito nítida. Poder-se-ia dizer que isto decorre tanto pelas condiçōes de saúde e de vida satisfatórias como da qualidade de assistência pré-natal, ao parto e ao recém-nascido.

Já em paises em desenvolvimento, como por exemplo, no Brasil, tem-se verificado nos últimos tempos que, apesar de partir de niveis elevados, tanto de mortalidade perinatal como materna, ao invés de uma rápida queda desses coeficientes assiste-se a uma redução de valores muito menos perceptiveis criando, portanto, uma situação paradoxal que no presente trabalho pretende-se discutir.

Assim, através de dados oficiais disponiveis será feita uma análise, tanto da assistência oferecida à gestante e ao parto como de relações entre as características dessa assistência com a mortalidade materna, perinatal, neonatal e natimortalidade no Estado de São Paulo.

\section{ASSISTÊNCIA PRÉ-NATAL}

Para uma avaliação real da assistência pré-natal no Estado de São Paulo há o grande obstáculo da falta de informação. Oficialmen-

\footnotetext{
* Departamento de Saúde Materno-Infantil da Faculdade de Saúde Pública da Universidade de São Paulo - Av. Dr. Arnaldo, 715 - 01255 - Såo Paulo, SP - Brasil.

* Associaça Hospital de Cotia - Av. Dr. Odair Pacheco Pedroso, 171 - 06700 - Cotia, SP - Brasil.
} 
te, conta-se com poucas fontes dè registros específicos do assunto. Os dados disponíveis, por sua vez, limitam-se a analisar quantitativamente a assistência. Sabendo-se que o pré-natal é uma atividade rotineira, sujeita a simplificaçōes, nem sempre desejáveis, a qualidade com que é realizado pode ser o fator determinante de sua efetividade.

Apesar disso, os números oficiais podem indicar tendências que refletem o comportamento da assistência pré-natal no Estado. O período mais recente que pode ser analisado são os cinco anos entre 1980 e 1984, cujos dados encontram-se divulgados pela Fundação SEADE', definindo uma tendência que se pode extrapolar para a atualidade, com margem segura de risco.

Excetuando-se uma pequena parcela da população que tem acesso à assistência pré-natal em consultórios particulares, as gestantes em São Paulo recebem-na nos serviços públicos: ou no Instituto Nacional de Assistência Médica e Previdência Social (INAMPS) (e seus conveniados) ou nos Centros de Saúde da rede pública da Secretaria da Saúde e de algumas prefeituras municipais.

Apenas os serviços da Secretaria da Saúde contam com programação específica de assistência pré-natal. Os serviços do INAMPS têm registro apenas de consultas de clínica obstétrica, permanecendo a dúvida do quanto representam de assistência pré-natal de fato. Pode-se afirmar apenas que são consultas dispensadas a mulheres grávidas ou puérperas, mas não organizadas dentro de um programa de assistência. Os serviços ligados às municipalidades e por elas gerenciados têm em comum a sua distribuição irregular no território do Estado (em geral nas prefeituras com maior disponibilidade financeira) e a heterogeneidade na abordagem que dão à questão da assistência pré-natal, além da quase absoluta falta de dados de produção que possam ser computados em conjunto com os da Secretaria de Saúde e do INAMPS.

A comparação entre os números das duas entidades mostra que o número de consultas de clínica obstétrica do INAMPS é sempre superior ao número de consultas de pré-natal da rede pública, numa razão de 7 para 2, mesmo com a queda da participação das consultas obstétricas no montante do movimento ambulatorial do INAMPS, observado neste período (Tabela 1).

Ainda com os mesmos dados, calculando-se artificialmente uma cobertura de $100 \%$ às gestantes no Estado de São Paulo, e somando o número de consultas dadas pela Secretaria da

\section{TABELA 1}

Número de consul tas obstétricas do INAMPS e Secretaria Estadual de Saúde de São Paulo. 1980-1984.

\begin{tabular}{ccc}
\hline & \multicolumn{2}{c}{ Número de Consultas } \\
Ano & INAMPS & Estado (S.E.S.) \\
\hline 1980 & 1.667 .691 & $\ldots$ \\
1981 & 1.771 .365 & 517.465 \\
1982 & 1.746 .894 & 611.198 \\
1983 & 1.721 .421 & 620.621 \\
1984 & 1.532 .279 & 449.440 \\
\hline
\end{tabular}

Fonte: Anuário Estatístico do Estado de São Paulo ${ }^{1}$

Saúde com as dadas pelo INAMPS, haveria uma concentração de 3 consultas por gestante. Apesar de baixa, a concentração de 3 consultas por gestante do Estado revela uma razoável eficiência em termos de infra-estrutura de atendimento, já que os pressupostos foram ideais. Este fato reforça a idéia de que a questão qualitativa é bem mais relevante do que a quantitativa no que tange à assistência pré-natal em São Paulo. Cabe salientar que não estão computados os dados dos serviços municipais. Possivelmente, adicionadas à sua parcela, alcançar-se-ia o número considerado desejável, internacionalmente, de 5 consultas por gestante ${ }^{15}$.

O pré-natal da rede pública mostra-se deficiente no periodo analisado, com baixos índices de cobertura e concentração. A maior cobertura foi de $21 \%$ e a maior concentração de 4,01 consultas por gestante na série histórica (Tabela 2).

\section{TABELA 2}

Percentagem de cobertura e número de consultas por gestaçäo das gestantes inscritas na rede da Secretaria Estadual de Sáde de São Paulo. 1980-1984.

\begin{tabular}{ccc}
\hline Ano & $\begin{array}{c}\text { Percentagem } \\
\text { de } \\
\text { Cobertura }\end{array}$ & $\begin{array}{c}\text { No de Consul- } \\
\text { tas por } \\
\text { Gestação }\end{array}$ \\
\hline 1980 & 17,22 & $\ldots$ \\
1981 & 18,38 & 3,68 \\
1982 & 20,41 & 3,83 \\
1983 & 21,65 & 3,91 \\
1984 & 16,18 & 4,01 \\
\hline
\end{tabular}

Fonte: Anuário Estatístico do Estado de São Paulo ${ }^{1}$

A melhora na captação precoce da gestante para o pré-natal na rede pública, em 1984 , não ultrapassou a $5 \%$ das inscrições no primeiro trimestre, ocorrendo também decréscimo idêntico nas inscriçōes no terceiro trimestre.

A PNAD (Pesquisa Nacional por Amostra Domiciliar) ${ }^{13}$, coordenada pelo IBGE, em 1981, 
TABELA 3

Percentagem de mulheres residentes em situação urbana por número de atendimentos pré-natais segundo as classes de rendimento mensal familiar per capita. Estado de São Paulo. 1981.

\begin{tabular}{|c|c|c|c|c|c|c|}
\hline \multicolumn{2}{|c|}{ Rendimento mensal per capita } & \multicolumn{5}{|c|}{ No de atendimentos pré-natais } \\
\hline (Salário mínimo - S.M.) & Nenhum & 1 & 2 a 5 & 6 ou + & ig & Total \\
\hline $\begin{array}{l}\text { Até } 1 / 4 \text { do SM* } \\
1 / 4-1 / 2 S M \\
1 / 2=1 \mathrm{SM} \\
+ \text { de } 1 \mathrm{SM} \\
\text { Ignorado }\end{array}$ & $\begin{array}{r}18,8 \\
13,7 \\
5,4 \\
0,8 \\
-\end{array}$ & $\begin{array}{l}4,1 \\
1,9 \\
2,1 \\
1,0 \\
-\end{array}$ & $\begin{array}{l}29,5 \\
27,3 \\
24,7 \\
18,1 \\
22,2\end{array}$ & $\begin{array}{l}47,6 \\
57,1 \\
67,6 \\
79,7 \\
77,8\end{array}$ & $\begin{array}{l}\overline{-} \\
\overline{0,2} \\
0,4 \\
-\end{array}$ & $\begin{array}{l}100,0 \\
100,0 \\
100,0 \\
100,0 \\
100,0\end{array}$ \\
\hline Total & 5,8 & 1,8 & 22,6 & 69,5 & 0,3 & 100,0 \\
\hline
\end{tabular}

Fonte: Perfil estatístico de crianças e mães no Brasil (1981)

* Inclusive família sem rendimento

revelou dados mais precisos a respeito da abrangência da assistência pré-natal no Estado de São. Paulo, independente da agência prestadora do serviço. Por amostragem populacional, pesquisando em 15.086 domicílios, verificou-se que $69,5 \%$ das mulheres têm utilizado o pré-natal com 6 ou mais atendimentos por gestação (Tabela 3).

Os números aqui apresentados estão perto dos recomendados e, portanto, os coeficientes de natimortalidade, mortalidade materna e perinatal deveriam estar em valores próximos aos de países desenvolvidos, porém, isso não é o que se observa no Estado de São Paulo.

Para explicar essa aparente discrepância entre uma rede de pré-natal satisfatória (em termos quantitativos) e elevadas taxas de natimortalidade e mortalidade materna e perinatal, há que se duvidar da qualidade com que essa assistência é prestada. Apesar de não se dispor de uma avaliação recente dessa qualidade, já em 1975, Ciari Jr. e col. ${ }^{5}$ chamava a atenção para o problema.

É importante realçar que deve ocorrer uma integração entre o pré-natal, a assistência ao parto e ao recém-nascido para que a qualidade da assistência seja garantida. Não há, no Estado, qualquer exemplo numericamente importante de uma tal integração.

\section{ASSISTENCIA AO PARTO}

Todo o processo reprodutivo requer uma série de atenções especiais; dentre esses momentos destaca-se o parto. Nesse sentido, Briquet ${ }^{4}$, em 1939, já referia que "não se pode considerar a função parturição como isenta de perigo, pois só nos Estados Unidos onde a assistência médi- co-social é bastante difundida, determinou, em $1937,4,4 \%$ de óbitos maternos".

A preocupação com o momento do parto ao longo do tempo passou a ser constante, refletindo-se até nas programações de saúde, que passaram a preconizar para a assistência ao parto, profissionais especializados na área da obstetrícia $^{4}$.

No Brasil, em nível de definição de política de saúde, este fato ocorreu em 1975, na 5: Conferência Nacional de Saúdes, que estabeleceu como uma de suas metas: "atingir a cobertura de $50 \%$ da assistência técnica ao parto".

Apesar das novas definições de política de saúde, nenhum mecanismo foi criado para que ocorresse a integração entre os organismos que realizam a assistência pré-natal e ao parto. Este fato impediu que o processo reprodutivo fosse visto em sua totalidade, favorecendo a segmentação e a descontinuidade na atenção à saúde do binômio mãe-filho, culminando com um maior risco de adoecer e morrer deste binômio, o que pode ser evidenciado com os dados recentes da mortalidade materna e perinatal, como se verá mais adiante.

TABELA 4

Percentagem de partos ocorridos em hospitais no Estado e Município de São Paulo, 1981 a 1985.

\begin{tabular}{ccc}
\hline Ano & $\begin{array}{c}\text { Estado } \\
\%\end{array}$ & $\begin{array}{c}\text { Município } \\
\%\end{array}$ \\
\hline 1981 & 87,5 & 85,5 \\
1982 & 88,3 & 86,1 \\
1983 & 89,2 & 87,5 \\
1984 & 89,8 & 87,9 \\
1985 & 90,5 & 88,5 \\
\hline
\end{tabular}

Fonte : Estatística do registro civil ${ }^{7}$ (1981 a 1985) 


\section{TABELA 5}

Percentagem de mulheres residentes em área urbana que tiveram filhos em hospital no período de referência de 12 meses, por tipo de parto e segundo classes de rendimento mensal familiar per capita. São Paulo, 1981.

\begin{tabular}{lccc}
\hline & \multicolumn{3}{c}{$\begin{array}{c}\text { Tipo de parto das mulheres } \\
\text { residentes em área urbana }\end{array}$} \\
$\begin{array}{c}\text { Rendimento mensal } \\
\text { familiar per capita em SM }\end{array}$ & Normal & Cesariana & Ignorado \\
\hline Até 1/4 S.M.* & 72,5 & 27,5 & - \\
1/4 - 1/2 S.M. & 63,4 & 36,6 & - \\
1/2 - 1 S.M. & 60,2 & 39,8 & - \\
+ de 1 S.M. & 44,0 & 55,7 & 0,3 \\
Ignorado & 55,5 & 44,5 & - \\
\hline Total & 54,7 & 45,2 & 0,1 \\
\hline
\end{tabular}

Fonte: Perfil estatistico de crianças e mães no Brasil ${ }^{13}$

SM - Salário mínimo

* Inclusive famńlia sem rendimentos

No Estado de São Paulo, na década atual, a quase totalidade dos partos tem ocorrido em hospitais (Tabela 4). Destes partos, para o ano de 1981 , segundo a PNAD, $54,7 \%$ foram normais e $45,2 \%$ cesarianas.

Para este mesmo ano, dos partos ocorridos no Município de São Paulo, nas instituições próprias ou contratadas pelo INAMPS, $36,98 \%$ foram normais e $63,02 \%$ cesarianas.

Os dados da PNAD $^{13}$ mostram ainda que quanto maior é a renda mensal familiar per capita maior é a incidência de partos do tipo cesariana (Tabela 5), sugerindo que o tipo de parto está muito mais ligado a outras questoes que não às de saúde. Em outras palavras, grande parte das cesarianas realizadas provavelmente não tiveram indicação obstétrica.
Sendo a população mais pobre a que apresenta um maior risco de adoecer e morrer era de se esperar que esta população tivesse uma incidência mais elevada de cesarianas do que a população de classe alta e/ou média alta, porém não a níveis superiores a $20 \%$ que é o aceito, atualmente, pela clínica obstétrica.

Quanto ao tipo de instituição prestadora de assistência ao parto no Município de São Paulo, observou-se que quando o serviço é próprio do INAMPS $73,98 \%$ dos partos são normais e $26,02 \%$ são cesarianas contra $37,29 \%$ e $62,71 \%$ respectivamente, quando este é contratado por aquela instituição (Tabela 6). Em relação às demais regiōes do Estado, todas as instituições prestadoras de assistência são contratadas e se pode observar uma alta incidência de cesarianas realizadas nessas instituiçōes $(46,16 \%)$, cifras estas, porém, mais baixas do que as encontra-

\section{TABELA 6}

Número e percentagem de parto segundo tipo de serviço do INAMPS, no Município e Estado de São Paulo, 1981.

\begin{tabular}{|c|c|c|c|c|c|c|c|c|c|c|}
\hline \multirow{3}{*}{$\begin{array}{l}\text { Tipo } \\
\text { de } \\
\text { Parto }\end{array}$} & \multicolumn{4}{|c|}{ Próprio } & \multicolumn{4}{|c|}{$\begin{array}{l}\text { Serviços do INAMPS } \\
\text { Contratados }\end{array}$} & \multirow{2}{*}{\multicolumn{2}{|c|}{$\frac{\text { Total }}{\text { Est. SP. }}$}} \\
\hline & \multicolumn{2}{|c|}{ Munic. SP } & \multicolumn{2}{|c|}{$\begin{array}{c}\text { Demais reg. } \\
\text { do Estado } \\
\text { de SP. }\end{array}$} & \multicolumn{2}{|c|}{ Munic. SP. } & \multicolumn{2}{|c|}{$\begin{array}{c}\text { Demais reg. } \\
\text { do Estado } \\
\text { de SP. }\end{array}$} & & \\
\hline & Nọ & $\%$ & No & $\%$ & Nọ & $\%$ & No & $\%$ & No & $\%$ \\
\hline Normais & 3.360 & 73,98 & - & - & 59.036 & 37,29 & 156.878 & 53,84 & 219.274 & 48,27 \\
\hline $\begin{array}{l}\text { Cesaria- } \\
\text { nas }\end{array}$ & 1.182 & 26,02 & - & - & 99.269 & 62,71 & 134.495 & 46,16 & 234.946 & 51,73 \\
\hline Total & 4.542 & 100,00 & - & - & 158.305 & 100,0 & 291.373 & 100,0 & 454.220 & 100,0 \\
\hline
\end{tabular}

Fonte: INAMPS. Dados brutos, não publicados. 
das na Capital do Estado. Este fato não é observado somente no Estado de São Paulo mas no Brasil como um todo, assim como relatam Barros e col. ${ }^{3}$, por exemplo.

Esses dados revelam que deve estar ocorrendo, principalmente nos serviços contratados pelo INAMPS, distorção na assistência ao parto por motivos econômicos e outros não relacionados à saúde da mãe e da criança propriamente dita, influenciados também pela política de pagamento feita por aquela instituição. Ao se confrontar esses índices, com os descritos em literatura, observa-se que são dos mais elevados do mundo, pois em países desenvolvidos essas cifras não ultrapassam a $20 \%$, como por exemplo, nos EUA é de $17,9 \%^{14}$, Suécia $11,9 \%{ }^{12}$, entre outros.

Era de se esperar que, com a quantidade de assistência pré-natal, o elevado número de partos ocorridos em hospitais e com o tipo de assistência ao parto encontrados no Estado de São Paulo, a morbimortalidade materna e perinatal tivessem atingido valores bastante baixos.

Os dados que serão aqui analisados não evidenciam este fato.

\section{MORTALIDADE MATERNA}

Ao se analisar os dados de óbitos de mulheres em idade fértil, ocorridos por complicações da gravidez, parto e puerpério para o Estado de São Paulo, pôde-se observar que o coeficiente de mortalidade materna (CMM), em $1980^{18}$, foi de 5,54 por dez mil nascidos vivos (nv) e, em 1984, de 4,86 por dez mil nv, taxas estas elevadas ao se comparar, por exemplo, com o CMM da Suécia, que entre os anos de 1971 a 1980 foi de 0,66 por dez mil nv8.

Ao se estudar a tendência desse coeficiente nos últimos 8 anos para o Estado e Municipio constata-se que houve uma ligeira redução dessa taxa, tanto para um como para o outro, porém esta não foi tão elevada que pudesse refletir uma mudança no perfil de saúde da população.

As principais causas de óbitos maternos são as relacionadas diretamente com a gravidez ( $78,3 \%$ no Estado de São Paulo e $80,9 \%$ no Município para 1983), seguidas do aborto $(13,2 \%$ e $11,8 \%$, respectivamente) e, por fim, as causas obstétricas indiretas $(8,5 \%$ e $7,3 \%$, respectivamente, para o mesmo ano).

Ao se estudar detalhadamente cada causa de óbito observa-se que as síndromes hipertensivas são a primeira causa de óbito materno, seguidas das sindromes hemorrágicas e do aborto (Tabela 7).

Dentre os óbitos por causas hipertensivas, nos anos estudados, a eclâmpsia aparece em cerca de $70 \%$ destes, a pré-eclâmpsia ao redor

\section{TABELA 7}

Principais causas de óbito de mulheres por complicação da gravidez, parto e puerpério, estado de São Paulo, 1980 e 1984.

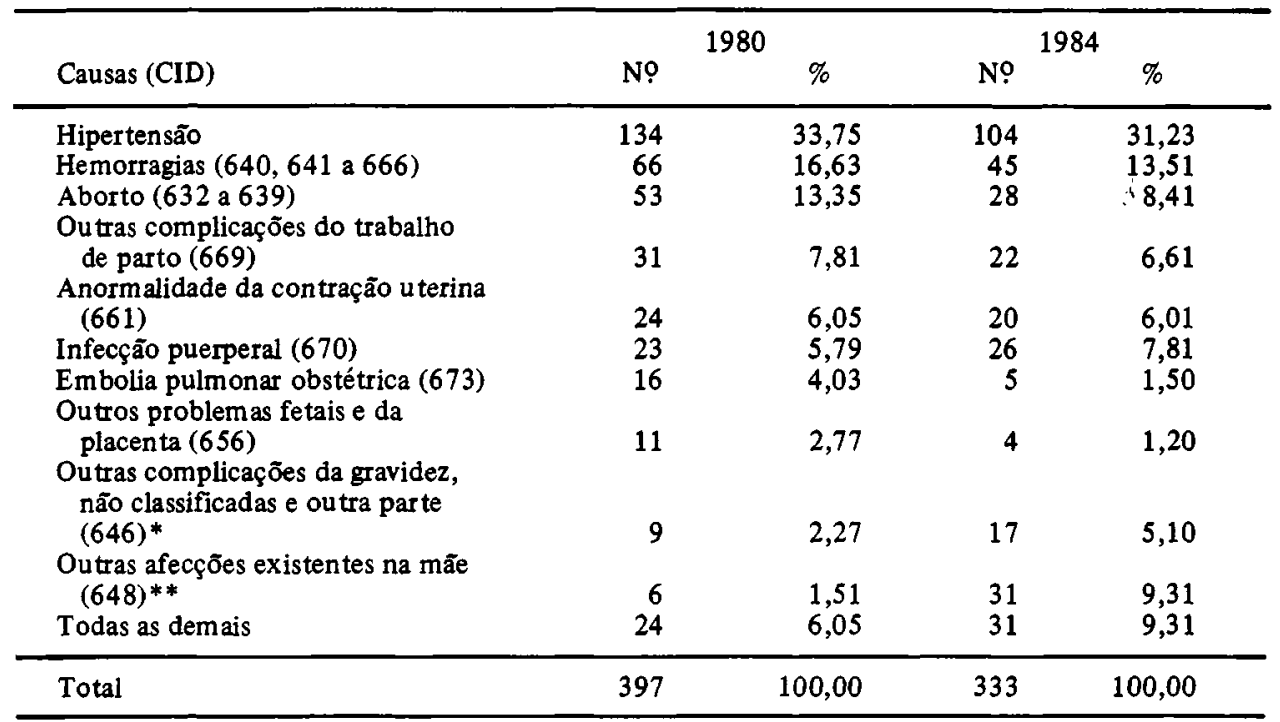

Fonte: Fundação SEADE - Dados brutos não publicados.

CID: Classificação Internacional de Doenças ${ }^{11}$.

* Edema ou ganho de peso excessivo sem hiper tensão, doença renal não específica sem menção de hipertensão, infecção urinária, transtorno de fígado.

** Diabetes Mellitus, anemia, doenças cardiovasculares. 
de $15 \%$ e as demais causas hipertensivas no restante dos casos.

Esses dados por si só refletem a má qualidade da assistência ao pré-natal, uma vez que a moléstia hipertensiva específica da gravidez pode ser diagnosticada e tratada neste momento, evitando-se assim evoluir para quadro mais grave (eclâmpsia) e, assim, para a morte.

Em relação às causas hemorrágicas, cerca de $55 \%$ dos casos são devidos ao descolamento prematuro da placenta, $30 \%$ a hemorragias pós-parto e o restante $(15 \%)$ a hemorragias do pré-parto. $O$ estudo dessas causas de óbito indica que a qualidade da assistência ao parto parece apresentar falhas, visto que esses óbitos poderiam, em sua maioria, ser evitados com uma atenção adequada neste período. Se se associar esta causa de óbito com a alta incidência de cesarianas, este dado se torna ainda mais alarmante, pois reflete o tipo de atenção que as gestantes estão recebendo no momento do parto.

Quanto a terceira causa de óbito, foi impossível estudá-la, pois não havia dados suficientes sobre 0 aborto.

De um modo geral, enquanto, por exemplo, nos EUA $^{9}$ a primeira causa de óbito materno é por causas obstétricas indiretas, em São Paulo a predominância é das causas obstétricas diretas que, seguramente, em sua maioria, seriam evitáveis.

Num estudo detalhado feito pelo Centro da OMS para Classificação de Doenças em Português $^{10}$, sobre mortes de mulheres de 10 a 49 anos no Município de São Paulo, para o ano de 1986, observou-se um sub-registro de óbitos maternos da ordem de $50 \%$, isto é, do total de óbitos maternos apenas metade foram registrados como tais; os demais só foi possível detectar pelas entrevistas domiciliares e estudo de prontuários.

Através da análise de cada caso pôde-se constatar que $34,7 \%$ dos óbitos foram devidos a causas obstétricas diretas, $26,3 \%$ a obstétricas indiretas e $40 \%$ a causas não relacionadas.

Observou-se, também, que $10,7 \%$ das mortes por complicaçōes da gravidez, parto e puerpério ocorreram após 42 dias do parto. Este fato faz com que esses casos não sejam computados como óbitos maternos, apesar de o serem, pois existe regra para a classificação do óbito neste sentido, o que impede o conhecimento real da magnitude deste problema.

Um dos fatos mais importantes revelados por essa pesquisa, diz respeito ao tipo de assistência recebido pelas mulheres grávidas, pois em cerca de $30 \%$ dos casos houve forte suspeita de imperícia ou negligência médica ou negligência da instituição prestadora de assistência.
Esses dados revelam que o abuso da tecnologia mais a imperícia e negligência, quer médica ou institucional, impõem à população de mulheres grávidas um maior risco de morrer por condições que quase com certeza poderiam ter sido evitadas.

\section{MORTALIDADE PERINATAL}

Os progressos tecnológicos na obstetrícia na última década levaram, em âmbito mundial, a melhoria da qualidade do diagnóstico dos problemas de saúde que afetam o concepto e como conseqüência houve aumento e melhor indicação de cesarianas. Esse aumento que, apesar de significativo, levou a taxas de cesarianas em geral inferiores a $20 \%$ do total de partos, contribuiu para uma evidente queda na mortalidade perinatal nos países desenvolvidos.

Ao contrário, em nosso meio, o aumento na incidência de cesarianas vem ocorrendo, nos anos 70 e 80 , em niveis assustadores e pouco contribuiu para uma diminuição da mortalidade perinatal. Este coeficiente, que para 1981 era de 33,47 por mil nascimentos, passou para 29,18 por mil em 1984 representando uma queda de apenas $13 \%$.

Comparando-se esses dados com os que se observa em países desenvolvidos, vê-se que, nesse período, mesmo sendo a mortalidade bem mais baixa (e, portanto, mais difícil de cair), apresentou quedas bem mais importantes, de tal forma que a presente mortalidade no Estado de São Paulo é cerca de duas a três vezes maior que a daqueles países.

Se a avaliação quantitativa dos serviços não proporciona uma explicação satisfatória para as precárias condições de saúde perinatal, tem-se que buscá-la na qualidade da assistência prestada, bem como nas condições de vida da própria população assistida.

Talvez a expressão mais direta das condições de vida da população, no que se refere ao período perinatal, seja a incidência de baixo peso ao nascer.

No Municipio de São Paulo (e talvez na Grande São Paulo), mais de $9 \%$ dos nascimentos são de crianças de menos de $2.500 \mathrm{~g}$. Apesar de não haver dados mais abrangentes sobre 0 interior do Estado, é provável que a incidência de recém-nascidos de baixo peso seja menor.

É notória a forte associação negativa entre peso ao nascer e mortalidade perinatal. $O$ que nem sempre é dito é que essa relação é muito mais forte quando os recém-nascidos de baixo peso são pré-termos ou pré-termos pequenos para a idade gestacional (PIG). 
Diversos estudos, seja realizados em nosso meio, como o de Areno $^{2}$, seja em outras populações ${ }^{16}$, têm mostrado que a incidência de prematuridade varia pouco, em torno de 3,5 a $5 \%$ do total de nascimentos. O que excede esses valores pode ser considerado tratar-se de recém-nascidos a termo, pequenos para a idade gestacional.

Apesar de maior que a dos recém-nascidos de termo, adequados para a idade gestacional, a mortalidade dos pequenos para a idade gestacional a termo é muito menor que a dos pré-termo e pré-termo pequenos para a idade gestacional. No caso do Estado de São Paulo, portanto, essa maior incidência de recém-nascidos de baixo peso não deve ser a principal razão para se ter tal mortalidade.

Desta maneira, ao se analisar os dois componentes da mortalidade perinatal, isto é, os natimortos e os óbitos de menores de 7 dias, observa-se que cerca de $40 \%$ dos óbitos correspondem a crianças nascidas mortas, enquanto que $60 \%$ ocorreram no período neonatal precoce ${ }^{1}$, no Japão, para $1984^{6}$, por exemplo, os óbitos corresponderam a $67,19 \%$ e $32,81 \%$, respectivamente. Isto é, além deste coeficiente ser numericamente muito mais baixo, o componente mais forte na mortalide perinatal é o número de natimortos, enquanto no Estado de São Paulo ainda o neonatal precoce exerce grande influência.
Estudando as causas de mortes neste período, chama a atenção o elevado número de causas perinatais tanto entre os natimortos como os óbitos de menores de 7 dias (Tabela 8). O segundo grupo de causas é referente a anomalias congênitas, seguido das doenças respiratórias agudas, sendo o quarto grupo de causas referente a doenças infecciosas e parasitárias.

Analisando detalhadamente estes 4 primeiros grupos de causas, apenas em relação aos óbitos de menores de 7 dias*, pode-se observar que, entre as causas perinatais, $61,6 \%$ dos óbitos foram por hipóxias e asfixias intra-uterinas, síndrome de angústia respiratória e síndrome de aspiração maciça, reflexo direto da assistência ao pré-natal, ao parto, e porque não dizer, de abuso das cesarianas (Tabela 9). Como se pode observar na Tabela 9, a segunda causa foram as infecções específicas que acometem o recém-nato, o que indica a qualidade de assistência ao recém-nascido. Nota-se também que a prematuridade e o baixo peso contribuem para apenas $16,6 \%$ dos obitos dentre as causas perinatais.

Apesar do tocotraumatismo corresponder a pouco mais de $1 \%$ de óbitos neste grupo, é uma das principais causas de morbidade do recém-nascido. Em estudo realizado por Tana$\mathbf{k a}^{19}$ em município da Grande São Paulo, ela correspondeu a cerca de $9 \%$ das morbidades apresentadas pelos recém-natos.

TABELA 8

Principais óbitos perinatais por causa no Estado e Município de São Paulo, 1984.

\begin{tabular}{|c|c|c|c|c|c|c|}
\hline \multirow[b]{2}{*}{ Óbitos (CID) } & \multicolumn{3}{|c|}{ Município } & \multicolumn{3}{|c|}{ Estado } \\
\hline & $\begin{array}{l}\text { Nasc. } \\
\text { mortos } \\
(\%)\end{array}$ & $\begin{array}{c}\text { Obitos } \\
<7 \text { dias } \\
(\%)\end{array}$ & $\begin{array}{c}\text { Total } \\
(\%)\end{array}$ & $\begin{array}{l}\text { Nasc. } \\
\text { mortos } \\
(\%)\end{array}$ & $\begin{array}{c}\text { Obitos } \\
<7 \text { dias } \\
(\%)\end{array}$ & $\begin{array}{l}\text { Total } \\
(\%)\end{array}$ \\
\hline $\begin{array}{l}\text { XV - Alg. Afeções Orig. no periodo } \\
\text { perinatal }\end{array}$ & 96,2 & 86,4 & 90,0 & 95,2 & 84,3 & 89,5 \\
\hline XIV - Anomalias Congênitas & 3,4 & 8,0 & 6,3 & 4,0 & 8,3 & 6,6 \\
\hline VIII - D. Apar. Respiratório & - & 4,0 & 2,5 & - & 4,0 & 2,4 \\
\hline I - D. Infec. e Parasitárias & 0,6 & 0,5 & 0,4 & 0,7 & 0,5 & 0,6 \\
\hline $\begin{array}{l}\text { III - D. Gland. Endoc. Nutr. Metab. e } \\
\text { Transt. Imunitários }\end{array}$ & - & 0,3 & 0,2 & - & 0,3 & 0,2 \\
\hline IX - D. Apar. Digestivo & - & 0,1 & 0,0 & - & 0,3 & 0,2 \\
\hline Demais causas & 0,0 & 0,7 & 0,5 & 0,0 & 1,1 & 0,5 \\
\hline Total & $\begin{array}{c}100 \\
(2272) \\
(36,8)\end{array}$ & $\begin{array}{c}100 \\
(3910) \\
(63,2)\end{array}$ & $\begin{array}{c}100 \\
(6182) \\
(100,0)\end{array}$ & $\begin{array}{c}100 \\
(7982) \\
(40,4)\end{array}$ & $\begin{array}{c}100 \\
(11790) \\
(59,6)\end{array}$ & $\begin{array}{c}100 \\
(19772) \\
(100,0)\end{array}$ \\
\hline
\end{tabular}

Fonte: Fundação SEADE - Dados brutos, não publicados.

* Classificação Internacional de Doenças 11

* Análise feita apenas em relação aos menores de 7 dias, pois não foi possível conseguir a causa detalhada de mortes dos natimortos para o Estado. 
TABELA 9

Percentagem de óbitos de menores de 7 dias por algumas afecçōes originadas no período perinatal, Estado de São Paulo. 1984.

\begin{tabular}{|c|c|}
\hline Causas (CID)* & $\%$ \\
\hline $\begin{array}{l}\text { Outras afecçōes resp. do feto e do recém-nascido } \\
\text { (Sind. asp. maciça en tre ou tras }(770)\end{array}$ & 23,93 \\
\hline $\begin{array}{l}\text { Hipoxias intra-u terina e asfixia ao nascer (768) } \\
\text { Síndrome de angústia respiratória }(769) \\
\text { Transt. rel. gest. de curta duração (765) } \\
\text { Infecçōes específicas do periodo perinatal (771) } \\
\text { Afecçס̄es maternas ( } 760 \text { e 761) } \\
\text { Compl. de placenta, cordão e mem branas (762) } \\
\text { Traumatismo de parto (767) }\end{array}$ & $\begin{array}{r}19,63 \\
18,08 \\
16,59 \\
5,38 \\
3,56 \\
2,80 \\
1,02\end{array}$ \\
\hline Todas as demais causas $(763,764,766,772-9)$ & 9,01 \\
\hline
\end{tabular}

Fonte: Fundação SEADE - Dados brutos - não publicados

* Classificação Internacional de Doenças ${ }^{11}$

Entre as anomalias congênitas, que corresponderam a $6,6 \%$ dos óbitos perinatais, as do coração e do aparelho circulatório foram as principais anomalias seguidas das do sistema nervoso. Dentre a mortalidade neonatal especifica ela correspondeu a $1,5 \%$, valor este inferior ao encontrado em países desenvolvidos, ao redor de 3 a $4 \% \%^{17}$. Longe de representar uma vantagem, deve-se à insuficiência destes diagnósticos.

Quanto às doenças respiratórias agudas, a principal causa foi a pneumonia $(2,4 \%)$, correspondendo a $90 \%$ dos óbitos neste grupo. Essas mortes neonatais foram provavelmente pneumonias aspirativas ou quadros terminais de processos septicêmicos, tornando evidente, uma vez mais, a má qualidade de assistência recebida pelo recém-nascido, quer no parto como após o nascimento, bem como a contaminação hospitalar.

O grupo de causas de doenças infecciosas e parasitárias contribuiu em $0,6 \%$ de óbitos, sendo a doença diarréica a mais importante. Cabe ressaltar ainda a presença de óbitos por lues congênita neste grupo de causa.
A meningite apesar de ser classificada no grupo de causas do sistema nervoso e dos órgãos dos sentidos também esteve presente como causa de óbito em menores de sete dias.

Concluindo, pode-se dizer que ao lado de um ambiente hospitalar absolutamente hostil, tanto para a grávida como para o recém-nascido que ainda é um imunodeprimido, agrega-se a má qualidade de assistência ao pré-natal, parto e recém-nascido; essa situação agrava-se pela falta de integração entre os serviços de pré-natal e o hospital, levando a mãe, concepto e recém-nascido a um risco de vida maior do que o eventualmente apresentado.

Finalmente, cumpre esperar das autoridades responsáveis pela elaboração e condução das politicas de saúde, que ao colocar em prática a proposta da reforma sanitária e de um Sistema Unificado e Descentralizado de Saúde, que levem em conta a qualidade da assistência e a integração dos serviços como os verdadeiros alvos a serem atingidos. Só assim será possível resolver esse paradoxo. 
TANAKA, A.C. d'A. et al. [Maternal and perinatal health position in the State of S. Paulo, Brazil]. Rev. Saúde públ., S. Paulo, 23:67-75, 1989.

ABSTRACT: The assistance offered during pregnancy and labour as also to the newborn child, and its relationship to maternal and perinatal mortality in the State of S. Paulo in 1984, is analysed on the basis of official available data. With respect to prenatal care the number of visits per woman was considered to be "sufficient" though of doubtful quality. The proportion of cesarean sections was very high $(46.2 \%)$. Maternal mortality was found to be 4.86 deaths per 10,000 live births, but despite its being high, this figure is certainly too low and the correct figure is probably twice as high. The principal cause of maternal deaths is toxemia in pregnancy, followed by hemorrhage and abortion. Most of these deaths could have been avoided with care during pregnancy and labour. The rate of perinatal mortality was found to be 29.2 deaths per thousand births in 1984 . This figure is also very high. The analysis of the causes of death for this period showed that the disorders which arose during the perinatal period were responsible for 90 per cent of the total number of deaths. The main causes of death in this group were the intra-uterine hypoxias and anoxias, asphyxia, respiratory distress syndrome and massive aspiration syndrome. These data bring to light the poor quality of the care offered to this group. The authors trust that the new policy of the Decentralized and Unified System of Health will take the quality of care as much as the integration of services into consideration with a view to overcoming the precarious maternal and perinatal health situation in S. Paulo. health.

KEYWORDS: Prenatal care. Delivery. Maternal mortality. Perinatal mortality. Maternal and child

\section{REFERÊNCIAS BIBLIOGRÁFICAS}

1. ANUÁRIO ESTATÍSTICO DO ESTADO DE SÃO PAULO: 1980-1984. (Fundação SEADE). São Paulo, 1981-1985.

2. ARENO, F.B. Contribuição ao estudo da antropometria do recém-nascido. São Paulo, 1984. [Dissertação de Mestrado - Faculdade de Saúde Pública USP]

3. BARROS, F.C. et al. Why so many caesarean sections? The need for a further policy change in Brazil. Hith Pol. Plann., 1:19-29, 1986.

4. BRIQUET, R. Ostetricia normal. Rio de Janeiro, Ed. Freitas Bastos, 1939.

5. CONFERÊNCIA NACIONAL DE SAÚDE, 5a , Brasília, 1975. Anais. Brasília, Ministério da Saúde, 1975.

6. DEMOGRAPHIC YEARBOOK: 1985. (United Nations) New York, 1987.

7. ESTATÍSTICA DO REGISTRO CIVIL: 1981-1985. (Fundação IBGE). Rio de Janeiro, 1983, 1984, 1986.

8. HŐGBERG, U. Maternal deaths in Sweden, 1971-1980. Acta obstet. gynecol. scand, 65:161-7, 1986.

9. KAUNITZ, A.M. et al. Causes of maternal mortality in the United States. Obstet. Gynecol., 65:605-12, 1985.

10. LAURENTI, R. et al. Mortalidade de mulheres de 10 a 49 anos no Município de São Paulo (com ênfase à mortalidade materna): Relatório final, 1? parte. São Paulo, Centro Brasileiro de Classifjcação de Doenças, 1988. [Apresentado a Organização Panamericana de Saúde por conta do contrato apo 334/85 - mimeografado]
11. MANUAL da classificação estatística internacional de doenças, lesð̃es e causas de óbitos; 9? revisão. São Paulo, Centro Brasileiro de Classificação de Doenças, 1980.

12. NIELSEN, T.F. \& HÖKEYARD, K.H. Postoperative cesarean section morbidity: a prospective study. Amer. J. Obstet. Gynec., 146:911-6, 1983.

13. PERFIL estatístico de crianças e mães no Brasil: situação de saúde, 1981. Rio de Janeiro, Fundação IBGE, 1984.

14. PLACEK, P.J. et al. Cesarean section delivery rates: United States, 1981. Amer. J. publ. Hith, 73:861-2, 1983.

15. PREVENTING low birthweight. Washington, National Academy Press, 1985.

16. PUFFER, R. \& SERRANO, C.V. Caracteristicas del peso al nacer. Washington, D.C., Organizacion $\mathrm{Pa}$ namericana de la Salud, 1988. (Publ. Cient. 504).

17. SIQUEIRA, A.A.F. de Mortalidade neonatal e prematuridade. São Paulo, 1974. [Dissertação de Mestrado - Faculdade de Saúde Pública da USP]

18. SIQUEIRA, A.A.F. de el al. Mortalidade materna no Brasil, 1980. Rev. Saúde públ., S. Paulo, 18:448-65, 1984.

19. TANAKA, A.C. d'A. Saúde materna e saúde perinatal: relaçōes entre variáveis orgânicas, sócio-econômicas e institucionais. São Paulo, 1986. [Tese de Doutoramento - Faculdade de Saúde Pública da USP]

Recebido para publicação em 2/5/1988. Reapresentado em 26/10/1988. Aprovado para publicação em 28/10/1988. 